\title{
A New Approach in the Design of High-Risk Infusion Technology
}

\author{
Robert S. Murphy* and Steven J. Wilcox** \\ *Faculty of Engineering, Manukau Institute of Technology, Auckland, New Zealand \\ ${ }^{* *}$ Faculty of Advanced Technology, Glamorgan University, Pontypridd, UK
}

\begin{abstract}
The syringe infusion pump has been established as the instrument of choice for high-risk infusions, where potent drugs are often delivered at low rates of flow. However, numerous instances of unexpected flow error with consequent patient physiological impact have been reported. These include unwanted bolus delivery on release of line occlusion, dosage fluctuation due to pump height change and fluid reflux within the multiple pump installations now common in the Intensive Care Unit (ICU). This article examines the performance of a typical ICU syringe infusion pump and identifies mechanical compliance, inherent in commercial designs, as a source of flow error that should not be ignored by equipment designers. A prototype low compliance system is described and tested with performance compared to the conventional design, demonstrating advantages in terms of lower flow error.
\end{abstract}

Keywords: infusion pump design, syringe pump, flow accuracy

\section{INTRODUCTION}

Electro-mechanical intravenous therapy pump technology has evolved over the last forty years from roller clamp control of the fluid flowing from a suspended fluid bag to various forms of 'positive pressure' pump apparatus. Peristaltic pumps, of rudimentary design, were replaced by so-called 'volumetric' pumps. A 'volumetric' pump is one where a fixed volume of fluid will be forced forward towards the patient site by some means of mechanical actuator. Whilst the volume will be fixed, the rate at which it is processed into the patient site may vary with time depending on mechanical and other factors. The volumetric pump is used as the principle of operation of the 'syringe pump' where a fixed volume disposable syringe is operated on to dispense fluid in a regular flow manner in accordance with the electromechanical displacement of the syringe plunger.

*Address correspondence to Robert S Murphy, Manukau Institute of Technology, Faculty of Engineering, Private Bag 94006, Manukau City, Auckland, New Zealand. E-mail: murphy16480@hotmail.com. 


\subsection{Flow Accuracy and the 'High-Risk' Infusion}

The need for flow rate accuracy depends on the type of agent being administered. In this study, we considered the 'high-risk' category of infusion or Category 'A' [1] where excellent short-term and long-term accuracy are required.

\subsection{Long Term, Short Term Accuracy, and Short Half-Life Drugs}

The long-term flow rate error (e.g., measurement over an interval greater than one hour) will tend towards zero provided an accurate volume is dispensed over this interval, with any transient fluctuations averaging out. In some applications where the fluid is of long half-life such as insulin or heparin, transient fluctuation of flow will be of lesser importance.

However, for drugs such as vasoconstrictors and dilators (e.g., dopamine, dobutamine and epinephrine) where half-life may be measured in minutes, the shortterm accuracy - defined as the accuracy measured over an interval equal to or less than the half-life - must be good. As an example of a drug that imposes strict conditions for administration, we considered sodium nitroprusside, an agent frequently used postoperatively for coronary bypass surgery. This agent has a half-life of 30 to 180 seconds and the impact of dosage fluctuation, causing under or over infusion would be either hypertension - placing stress on sutures and the risk of internal bleeding, or undesired hypotension [2].

Exhaustive testing by national test houses, such as Bath Institute of Medical Engineering in the UK and others, has shown that only syringe infusion pumps can be recommended for high accuracy in the short-term [3].

\subsection{Measurement of Short Term Accuracy}

Typical flow rates in an intensive care unit (ICU) often range between $1 \mathrm{mlh}^{-1}$ and $5 \mathrm{mlh}^{-1}$ and the accurate delivery of such small volumes requires extremely stable apparatus. In order to approve equipment for use, the International Standard IEC 60601-2-24 [4] flow accuracy measurement technique is to digitally weigh fluid expelled from the pump system with respect to time and to compute the implied flow rate. In order to prevent external factors from influencing the measurements, the pumpunder-test is allowed to establish stable flow before commencing measurements. This protocol is extremely useful for demonstrating the mechanical fidelity of the actuator system and test results reported to national agencies are often made available in the public domain [5].

\subsection{Short-Term Accuracy Evaluated by 'Constancy Index'}

Data from the accuracy testing protocol can be processed to provide a 'constancy index' [5], which has been defined as the shortest time period over which measured flow rate does not exceed $+/-10 \%$ of the intended set (mean) flow rate. For example, a 'constancy index' of 5 minutes means that, in any 5 minute interval, the average flow rate is within $10 \%$ of the desired rate. This concept of a constancy index is recommended [5] for use by clinicians in selecting equipment appropriate for use with short half-life drugs such that the index should not be greater than the half-life. 
Whilst the standard flow accuracy testing methods using gravimetric measurement are a useful guide, they do not account for, or predict, flow accuracy in a dynamic environment.

\subsection{System Compliance}

System compliance is a ratio of change of volume due to change in pressure, viz. $\Delta \mathrm{V} / \Delta \mathrm{P}$. Pumps with lower compliance will be inherently more accurate during changes in environment. A low-compliance system would generate a much smaller change in volume with respect to change in pressure; hence, if the pump pressure cut-off point is unchanged, the bolus volume released after an occlusion alarm would be smaller for the low-compliance system. A low-compliance infusion pump would offer fast start-up, immunity from serious bolus flow on height change, speedy recognition of line occlusion, small bolus release on release of line occlusion and the ability to operate within multiple pump systems without generating or being subject to fluid reflux. Researchers have indicated (with the manufacture of a novel pump system - the Panomat-P10 $\left.{ }^{\circledR}\right)$ the need for a low compliance pump system in order to deliver accurately at low flow rates $[6,7]$.

Pump system mechanical compliance has been linked with various instances of unwanted flow delivery [8-16] with one research group detailing a practical realization of a pumping system using a unique design method [17] to reduce compliance and hence mitigate compliance-based flow errors [6,7]. This particular device offers a micro-volume rotary chamber system described by Patent [17] suitable for high accuracy dosing of small volumes.

\subsection{Objectives of the Study}

The first objective of this study was to demonstrate the unwanted flow characteristics of a typical critical care $50 \mathrm{ml}$ syringe pump and to determine whether this unwanted behavior could be associated with the mechanical design - specifically system compliance - by examining the mathematical basis for pump flow.

The second objective was to show that a simple mechanical prototype pump design using low compliance components would achieve significant improvement in low-flow pump performance and so validate a need for a reconsideration of manufacturing methods used for the infusion apparatus in intensive care.

\section{METHODS}

In this study, the flow characteristics of a typical commercial $50 \mathrm{ml}$ critical care syringe infusion pump were observed by noting, in particular, unsteady flow caused by operating conditions such as start-up of flow delivery and change in pump height. Equipment evaluation reports for various manufacturers' 50/60 ml pump systems, available in the public domain, suggested that the test syringe pump chosen was of typical performance for its type with respect to accuracy, start-up delay and susceptibility to height change. A set of equations suitable to describe pump flow were established to demonstrate a relation between undesirable flow error and the mechanical compliance of the infusion system. 
Finally, an alternative approach to infusion pump design was proposed seeking to minimize system compliance and improve flow accuracy during disturbances to the infusion system environment.

\subsection{Flow Rate Characteristics of a Typical Critical Care Syringe Pump}

The test apparatus consisted of a digital weighing platform with serial digital data output to a computer running a customized application program that processed sequential weight data from the platform. This arrangement (Figure 1) duplicated the apparatus of IEC 60601-2-24. The Standard specifies flow rate testing without detailing the type of administration set or extension line to be used. We chose a typical extension set - type Baxter ${ }^{\circledR}$ 'Minivolume Extension Set' 2C5687. In addition, we used an $18 \mathrm{~g}$ cannula, $1.2 \mathrm{~mm}$ as ISO 7864, detailed in the IEC 60601-2-24 Standard. The resolution of the digital weighing instrument was $0.0001 \mathrm{~g}$. We undertook measurements at a room temperature of $20^{\circ} \mathrm{C}$ with calculations based on fluid viscosity at this temperature. Long-term (over several hours) flow accuracy at low flow rates would normally require some mitigation of error due to fluid evaporation. Our use of the measurement apparatus was over short intervals where such evaporation error would be negligible. The weighing container and platform were within a glass containment vessel offering protection from any error due to transient air movement.

A typical commercial 50/60 ml syringe pump, rated as suitable for critical care applications, was tested. The syringe system was filled with water and the line set manually primed to expel air with the tip of the cannula beneath the water level within the measurement beaker as detailed by the Standard.

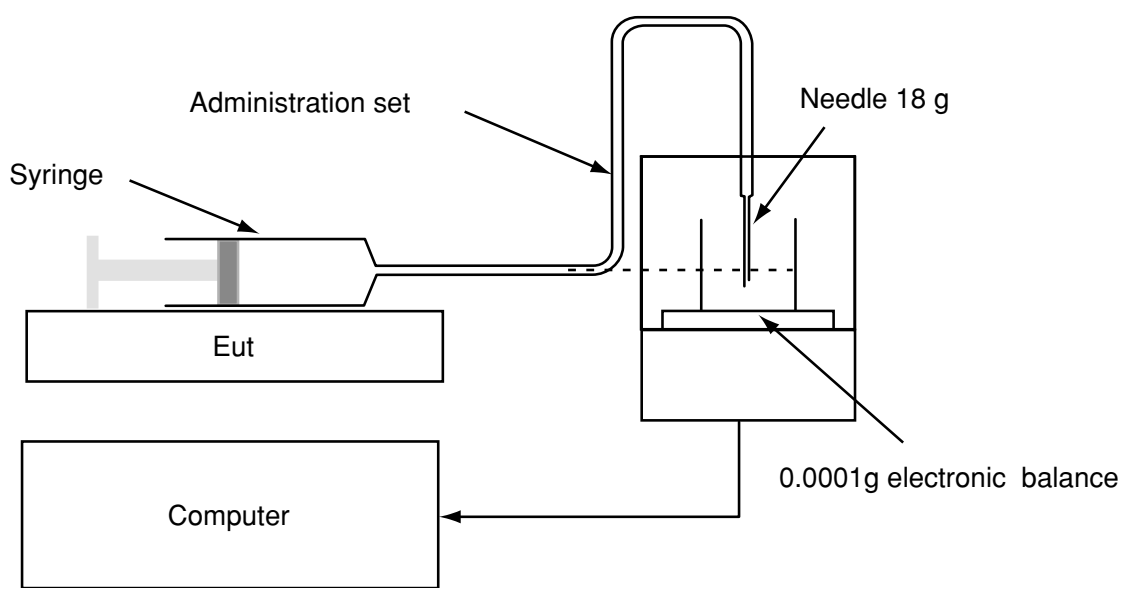

Figure 1. ISO 60601-2-24 flow accuracy test apparatus requiring the position of the syringe exit for the Equipment Under Test (EUT) to be at the same level as the fluid in the weighing container monitored by electronic digital balance and computer. 
We conducted three identical series of tests for start-up delay measurement - primed and unprimed - and susceptibility to change in pump height. The variation in start-up time (defined as the time taken to deliver within $+/-10 \%$ of the set rate) was found to be within $5 \%$ from experiment to experiment and any variation in the characteristic generated by height change was not measureable. The results presented represent those of the final series of tests performed.

\subsubsection{Start-Up Characteristic - Unprimed}

Many manufacturers offer advice to 'prime' the pump system before patient connection by running the pump without patient connection at an elevated rate for a short period of time until flow is seen to exit the cannula end. This is to remove any mechanical slack in the system so that, in theory, the set infusion will commence immediately after the patient connection is made and the pump is set to run. We first measured the characteristic time taken to start delivery within $+/-10 \%$ of the chosen flow rate, when a pump had been loaded with a fluid filled syringe and line set but without initial pump priming i.e., the unprimed test protocol.

\subsubsection{Start-Up Characteristic - Primed}

The flow characteristic of the pump was measured for an identical pump system that had been primed in the manner described. The 'primed' test protocol includes running the pump at elevated rate until regular flow is observed at the fluid system exit, halting the pump, setting the flow rate to the required test rate of $1 \mathrm{mlh}^{-1}$ before immediately commencing flow delivery at this chosen rate and commencing flow measurement timing.

\subsubsection{Influence of Height Change}

The pump system was allowed to establish regular flow, then raised in height relative to the weighing platform, and subsequently returned to the original height with observation being made of the consequent flow disturbance. The method used was the provision of a stable platform $30 \mathrm{~cm}$ above the reference pump location. At a time $160 \mathrm{~s}$ after the establishment of steady flow, the pump under test was raised manually to the higher platform. At time $390 \mathrm{~s}$ after the establishment of steady flow, the pump was removed from the platform back to the reference position. In each case, the change in height was accomplished within $2 \mathrm{~s}$.

\subsection{Effect of Mechanical Compliance on Flow Characteristics}

The relation between mechanical compliance of syringe pumps and unwanted flow characteristics has been recognized both during clinical use and by the establishment of a mathematical simulation model [8] based on equations (1) and (2). Non-turbulent flow in any system can be determined by use of Poiseuille's Equation:

$$
P_{2}-P_{1}=\frac{8 Q \eta L}{\pi R^{4}}
$$


where

$P_{2}-P_{1}=$ pressure gradient across the restriction length $(\mathrm{Pa})$.

$Q=$ volumetric flow rate $\left(\mathrm{m}^{3} \mathrm{~s}^{-1}\right)$.

$h=$ fluid viscosity Pa.s.

$L=$ restriction length $(\mathrm{m})$.

$R=$ average internal radius of the restriction formed by the line set and cannula (m).

In any system, flow into the system must equal flow out from the system, except in a system with mechanical compliance where the effect of change in system volume (compliance) will cause an additional unwanted error flow component. A simple mathematical model based on this non-ideal system has been shown to approximate the flow characteristic as a first order differential equation based on Equation (1) with a pressure change term added such that:

$$
Q=\left(P_{2}-P_{1}\right) A+C \frac{d P_{2}}{d t}
$$

where

$Q=$ volumetric flow rate $\left(\mathrm{m}^{3} \mathrm{~s}^{-1}\right)$.

$P_{2}=$ pump pressure $(\mathrm{Pa})$.

$P_{1}=$ patient pressure $(\mathrm{Pa})$.

$A=\pi R^{4} / 8 \eta L=$ system restriction $\left(\mathrm{m}^{3} \mathrm{~Pa}^{-1} \mathrm{~s}^{-1}\right)$.

$C=$ system compliance $\left(\mathrm{m}^{3} \mathrm{~Pa}^{-1}\right)$.

This relationship shows how short-term flow rate of the compliant system is influenced by system compliance $C$ and change in pressure $P_{2}$ (however caused) at the syringe exit.

Equation (2) states an easy to appreciate relationship - namely, that the flow rate $Q$ entering the pump system by mechanical displacement of the syringe plunger would be identical to the flow leaving the system via the extension line $\left(P_{2}-P_{1}\right) A$ under conditions of zero mechanical compliance, i.e. $C=0$. However, a non-zero value for $C$ results in an additional internal flow term that will act as a flow error component.

\subsection{An Alternative Design Approach}

In order to demonstrate the potential advantages of a low compliance system, a simple prototype was built using a conventional 2-ml BD Plastipak ${ }^{\circledR}$ disposable syringe and a motorized constant volume valve. This arrangement is shown in Figure 2. The syringe plunger was attached to the end of a Zaber NA11B30 (Zaber Technologies Inc.) precision linear actuator with actuation range of $3 \mathrm{~cm}$ and resolution of $3.6 \mu \mathrm{m}$, providing $80 \mathrm{~N}$ force at a maximum linear actuation rate of $20 \mathrm{~mm}$ per second. A generic plastic disposable three-way valve, as used in standard hospital practice to divert flow manually, was attached to a rotary motor (type Futuba ${ }^{\circledR}$ Standard Servo S3003) allowing 90 degrees of rotation to permit, in turn, the syringe outlet (port1, Fig. 2) to make a fluid pathway to the line set (port 3) to expel fluid to the patient site, or to the fluid reservoir (port 2) to allow the syringe to be filled. The system was set up to be a reciprocating pump such that fluid was input from the reservoir at a fast rate (completion of fill within $10 \mathrm{~s}$ ) followed by output to the extension line exit at the commanded slow rate chosen as $1 \mathrm{mlh}^{-1}$ in this experiment. 


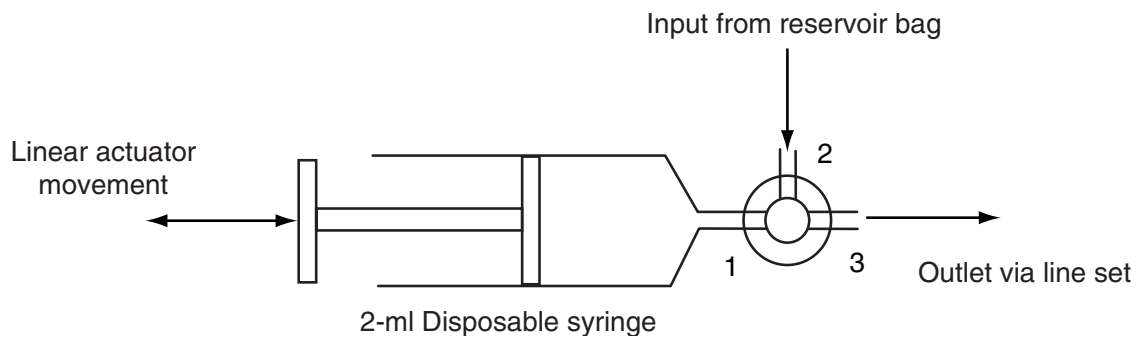

Figure 2. Prototype system using a linear-actuator-operated 2-ml syringe and rotary outlet valve.

\section{RESULTS}

In the following illustrations, gravimetric data had been sampled at 1-s intervals then applied to a 30-s running average filter. Figure 3 shows the unprimed start-up characteristic of a typical $50-\mathrm{ml}$ syringe pump. The flow rate remained near zero for about $1500 \mathrm{~s}$ before it started to increase asymptotically and reached the set rate of $1 \mathrm{mlh}^{-1}$ after $2300 \mathrm{~s}$. The start-up time (defined as the time taken to deliver within $+/-10 \%$ of the set rate) was seen to be approximately $2250 \mathrm{~s}$ from the curve. On the other hand, the flow rate test result of the present prototype system due to unprimed start-up at $1 \mathrm{mlh}^{-1}$ is exhibited in Figure 4.

The flow rate assumed the set rate of $1 \mathrm{mlh}-1$ within one minute of start. The startup time was seen to be about $20 \mathrm{~s}$, indicating a greatly improved response compared to the conventional 50-ml syringe pump in Figure 3. Even after 'priming', a typical 50-ml

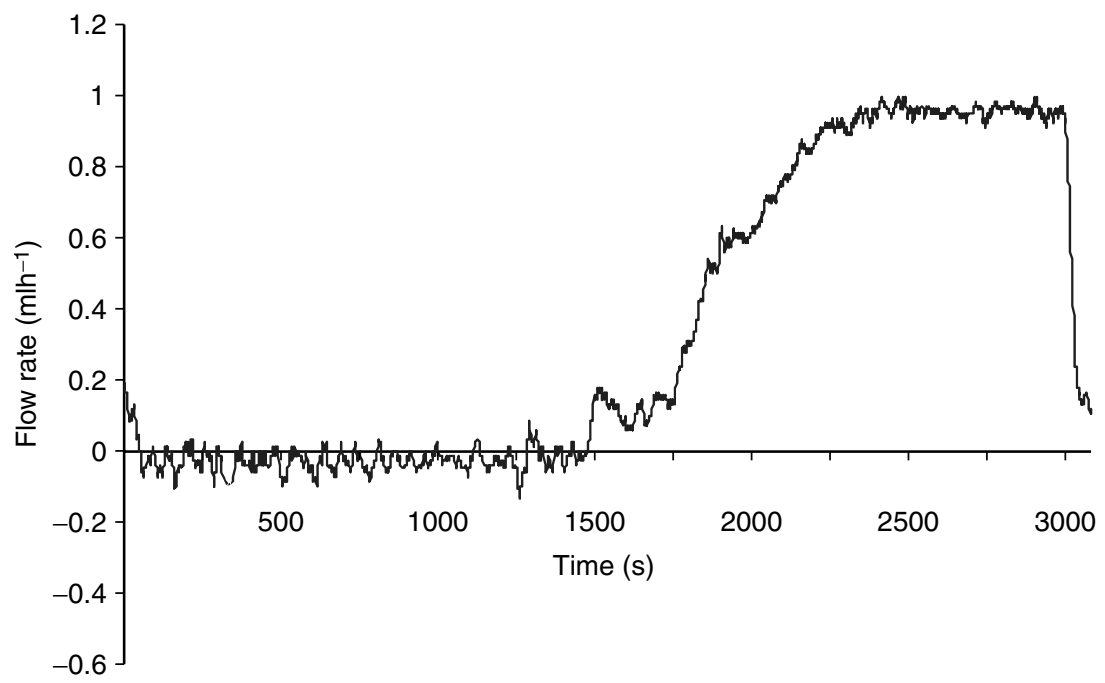

Figure 3. The flow data of a typical 50-ml syringe pump subjected to unprimed start-up at a set infusion rate of $1 \mathrm{mlh}^{-1}$. Range $0-3000 \mathrm{~s}$. 


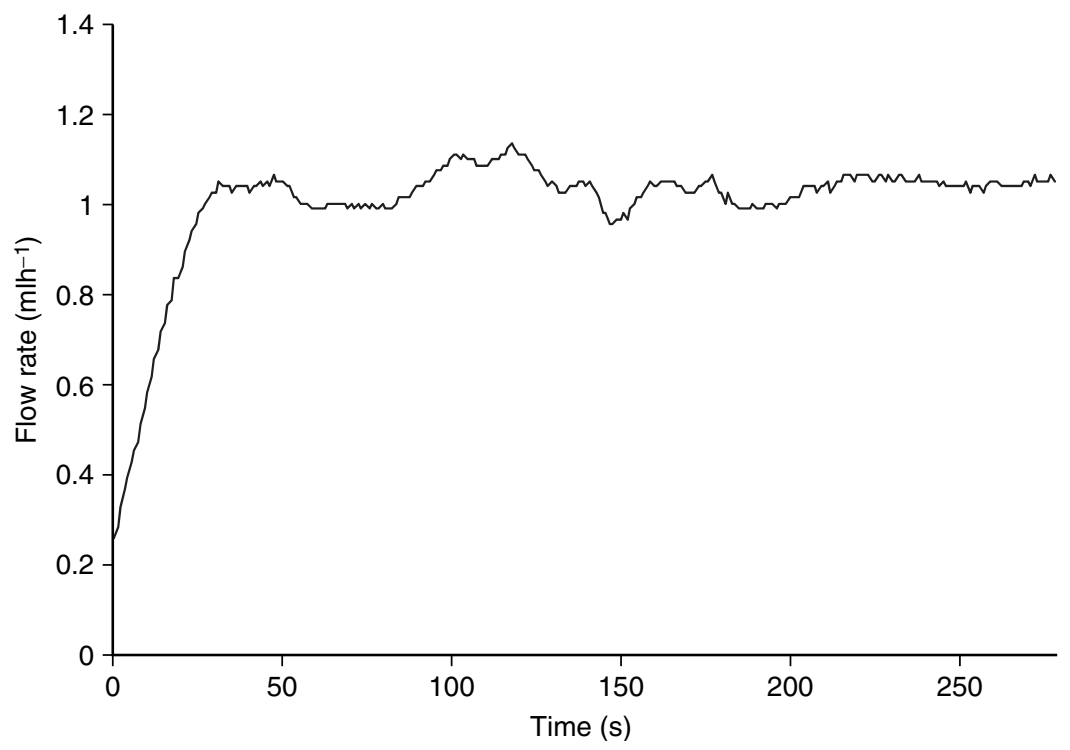

Figure 4. Flow rate of the prototype system due to unprimed start-up at $1 \mathrm{mlh}^{-1}$ showing delivery within $10 \%$ of set rate after about $20 \mathrm{~s}$. Range $0-250 \mathrm{~s}$.

syringe pump still exhibited a start-up time in excess of $500 \mathrm{~s}$, as shown by the curve in Figure 5.

The effect of height change on the flow rate of a typical 50-ml critical care pump is demonstrated in Figure 6. The pump was raised for $30 \mathrm{~cm}$ at time $160 \mathrm{~s}$, resulting in a

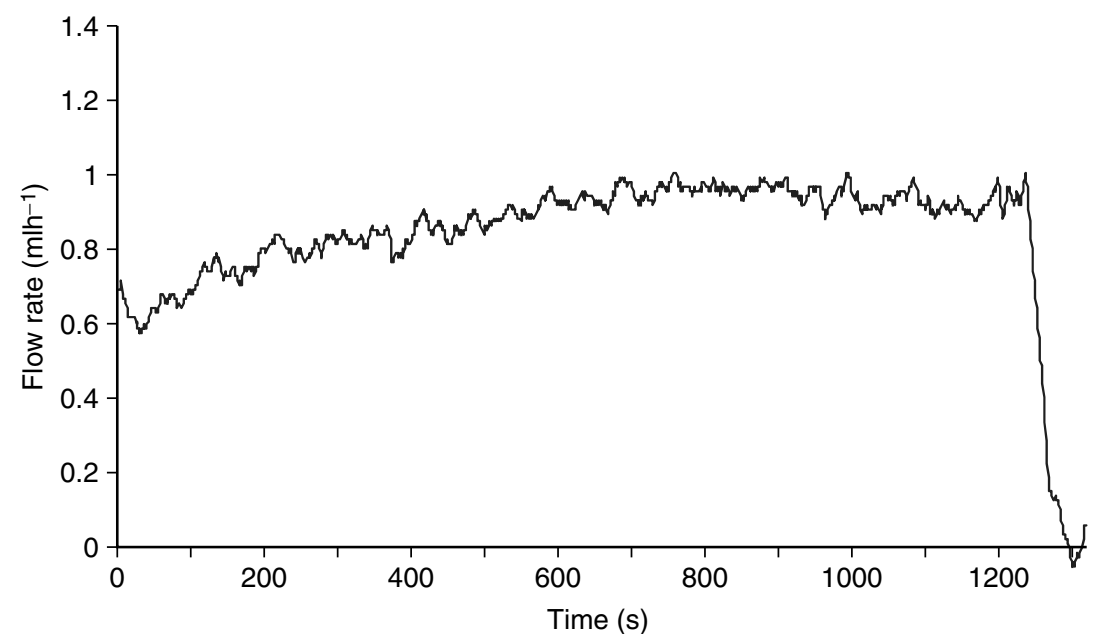

Figure 5. Primed start-up at $1 \mathrm{mlh}^{-1}$ of the typical 50-ml critical care syringe pump. Range $0-1300 \mathrm{~s}$. 


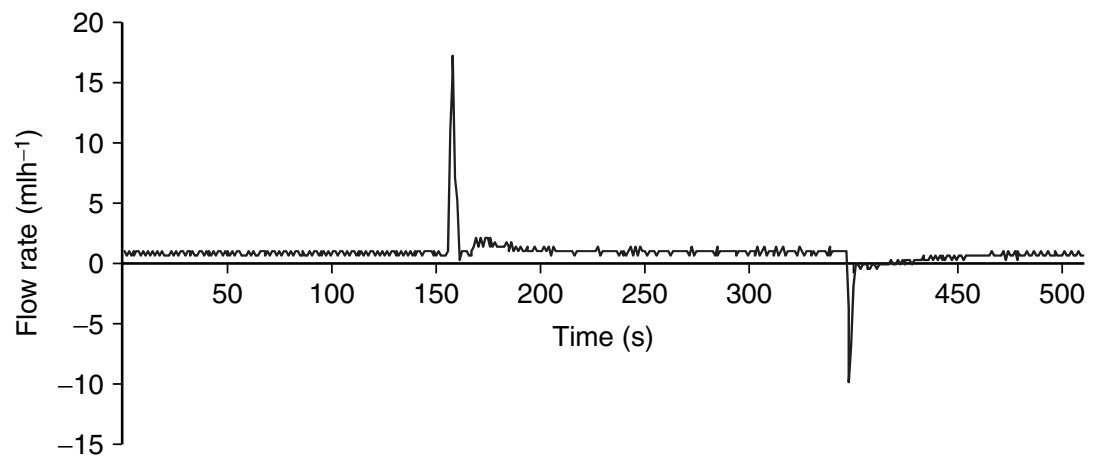

Figure 6. Flow disturbance with the typical 50-ml critical care syringe pump due to change in pump height.

surge of flow rate of about $17 \mathrm{mlh}^{-1}$, then, at time $390 \mathrm{~s}$, restored to its original height. The lowering of the pump resulted in negative flow followed by a period of zero flow. On the other hand, flow disturbance with the present prototype system due to height change is exhibited in Figure 7, where raising the pump height for $30 \mathrm{~cm}$ at about $160 \mathrm{~s}$ resulted in a positive bolus of about $7.5 \mathrm{mlh}^{-1}$, much smaller than that with a typical $50-\mathrm{ml}$ critical care syringe pump demonstrated in Figure 6, and no significant disturbance was observed when the pump was restored to its original height at time 390s. We subsequently discovered that the positive bolus was almost entirely due to the

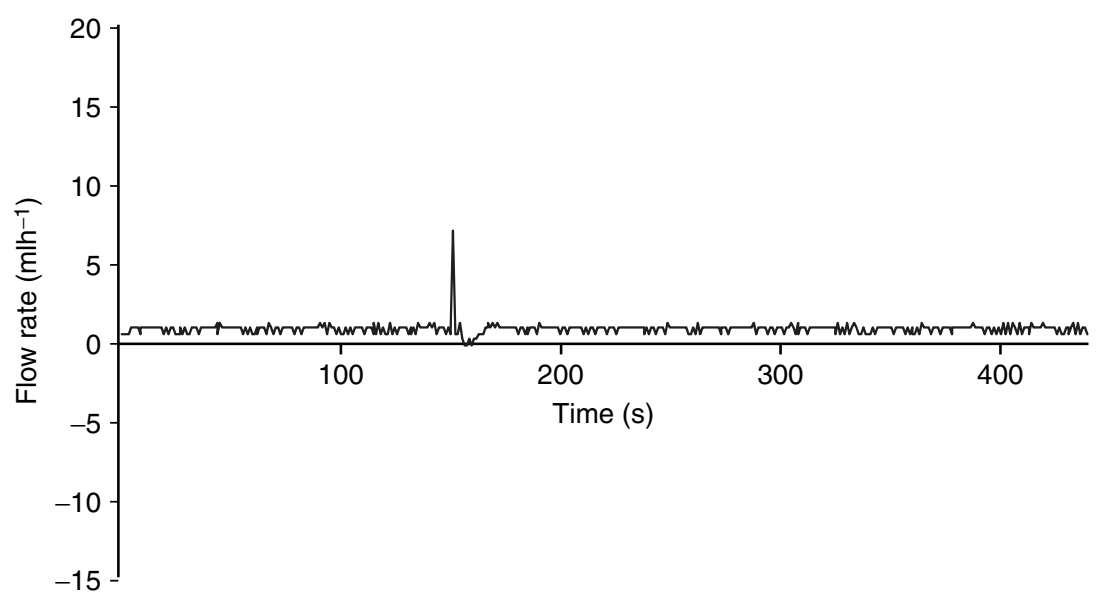

Figure 7. Flow disturbance with the present prototype system due to height change. Raising the pump height by $30 \mathrm{~cm}$ at time ca. $160 \mathrm{~s}$ resulted in a small positive bolus, but no disturbance was observed when the pump was restored to its original height at time $390 \mathrm{~s}$. Range 0-400 s. 
Table 1. Comparison of performance between the 50-ml typical syringe pump and the present prototype pump for start-up delay and change of height

\begin{tabular}{lll}
\hline Test & 50-ml syringe pump & Prototype pump \\
\hline *Start-up delay (unprimed) & ca. $2200 \mathrm{~s}$ & ca. $20 \mathrm{~s}$ \\
Start-up delay (primed) & ca. $500 \mathrm{~s}$ & $<20 \mathrm{~s}$ \\
Maximum $+/-$ peak flow & $+17 \mathrm{mlh}^{-1}$ to $-10 \mathrm{mlh}^{-1}$ & $+6 \mathrm{mlh}^{-1}$ to nil \\
$\quad$ error during height change & &
\end{tabular}

* Start-up delay defined as the time taken for flow to achieve a value within $10 \%$ of the set rate i.e. 1 mlh-1 in this experiment.

collapse in volume of the flexible Baxter® Minivolume Extension Set 2C5687 of 1.83 $\mathrm{m}$ length, and when this was replaced by an Alaris ${ }^{\circledR}$ coiled rigid plastic line set (Alaris ${ }^{\circledR}$ Impromediform GmbH Spiral Extension Set MFX1953), the positive bolus was not discernable within the noise threshold of the stable $1 \mathrm{mlh}^{-1}$ flow.

The results of start-up delay and flow rate disturbance due to height change are summarized in Table 1. The commercial 50-ml syringe pump exhibited prolonged startup delay compared to the present prototype for both primed and unprimed start conditions. The commercial pump exhibited pronounced transient flow error due to height change whilst the prototype flow error due to height change was much reduced.

\section{DISCUSSION}

The relation between the mechanical compliance of a syringe infusion pump and the ability to deliver accurate flow rate after change in the environment of the pump has been reported [8]. Numerous researchers [9-16] have identified environmental factors that cause flow error. These instances may, in some circumstances, be avoided by ensuring the infusion apparatus is maintained in a stable environment. However, flow error caused by change in pump height can also be caused by transient change in patient venous pressure [8].

Bolus release after line occlusion has been considered as an unwanted characteristic of infusion apparatus, leading to advice that pumps should be set to very low pressure alarm cut-off so as to minimize consequent bolus delivery. Users are advised not to change the pump height during infusion, but, in practice, this may be difficult to achieve when the patient is transferred by ambulance. Some manufacturers have tried to minimize the bolus release on occlusion by causing the driving mechanism to retract slightly after alarm cut-off to reduce pressure within the system and therefore the bolus. This solution, along with the care that may be taken in keeping equipment still, merely mask a design problem viz. system compliance.

We performed a number of tests based on the ISO (International Standards Organization) protocol for flow measurement equipment and methods [4]. These included measuring the 'unprimed' start-up characteristic of a typical 50-ml intensive care syringe pump (Figure 3) and demonstrated an unacceptably large delay before delivery 
within $10 \%$ of the set rate of $1 \mathrm{mlh}^{-1}$. Even when 'primed' (Figure 5), the flow rate was still in a condition of under delivery for an extended period of time. The slow start-up of delivery can be explained as the physical process of fluid flowing to 'inflate' the extracorporeal fluid circuit rather than flowing to the line exit and the patient.

In the case of a syringe infusion system comprising pump mechanism, syringe, extension line and cannula, the compliance will be small in terms of volume change. However, at the low flow rates common in ICU, i.e., $1 \mathrm{mlh}^{-1}$ to $5 \mathrm{mlh}^{-1}$, the consequence to flow accuracy, due to such tiny changes in the system volume, is appreciable.

Users are aware of the risk of bolus infusion when the height of a conventional pump is changed $[10,11]$, and this phenomenon was demonstrated in this study (Figure 6). Of particular note is a characteristic negative fluid bolus followed by a zero flow period when the pump was returned to the reference height. The zero-flow period - also commented on by other researchers [3] - was probably due to movement of the mechanical actuator relative to the syringe holder assembly such that mechanism 'backlash' must be taken up before flow can resume.

In a typical 50/60-ml syringe pump, system compliance is due to mechanical flexing of the actuator and loose fitting of the syringe within the pump, compliance due to distortion under pressure of the syringe and rubber plunger and finally the compliance of the extension set. For this type of system, a flexible extension line will be responsible for typically $20 \%$ of total system compliance [8] with the remainder due to syringe and pump mechanism.

This study would suggest that system compliance cannot be ignored as a contributory factor in flow error. If the system compliance were largely removed, the unwanted phenomena of flow error, caused by such factors as height change and start-up, would be mostly eliminated. In order to test this hypothesis, a simple prototype pump utilizing a 2-ml syringe, precision linear actuator and reservoir fluid bag was evaluated in the present experiment. The summarized results of Table 1 would suggest that a low mechanical compliance design does offer advantages in accurate flow delivery. The 'unprimed' start-up test when conducted on the conventional 50-ml syringe pump, had resulted in a delay in excess of 35 minutes (Figure 3), demonstrated a period of approximately 20 seconds before delivery within $10 \%$ of the commanded $1 \mathrm{mlh}^{-1}$ rate for the prototype system (Figure 4).

The 'height test', as detailed in this study, when applied to the prototype (Figure 7), demonstrated a much reduced positive bolus on height increase and no discernable negative bolus on returning the pump to the reference height.

The present prototype pump, using a 2-ml syringe and motorized flow directing valve - was intended to demonstrate that a system with minimization of compliance as part of the design would greatly reduce unwanted phenomena such as start-up delay and occlusion bolus and point towards a direction for future manufacturing development. Our experiment demonstrated promising results - reduced start-up delay in infusion and negligible flow error due to height change. This prototype would seek to complement and support the published findings of the Panomat-P10® researchers by associating observed flow error with a mathematical definition of system compliance. 
The prototype design described in this article would need further development in order to offer a practical pumping system. In particular, the manner of the mechanical location of the disposable items and the need to minimize the 'fill time', viz. the time taken to fill the syringe from reservoir before resuming normal flow, would need to be addressed. It is hoped that the concept of improving pump operational accuracy by the use of low compliance components, adopted by both the Panomat-P10® and our prototype, might persuade manufacturers to consider a new approach to design.

The ISO standard testing protocol for flow rate accuracy should be deemed as only an indication of the mechanical fidelity of the pump driving mechanism and should be considered along with measured compliance when evaluating a critical care infusion pump for use in normal operating conditions. A modification of the International Standard to measure, and stress the importance of, compliance as a specific parameter influencing flow accuracy might be useful.

\section{CONCLUSION}

Experiments were conducted to test the performance of a prototype pump system using a 2-ml syringe and motorized flow directing valve. Compared with a typical commercial syringe pump, the prototype system showed a shortened start-up delay for both primed and unprimed start conditions. The prototype also demonstrated much reduced flow error due to height change than the commercial pump. These results support that a system with minimized mechanical compliance as part of the design would greatly reduce undesirable behavior such as start-up delay and occlusion bolus.

\section{REFERENCES}

[1] Dougherty, L. and Lister, S.E., eds., The Royal Marsden Hospital Manual of Clinical Nursing Procedures, $6^{\text {th }}$ edn., Wiley-Blackwell, Hoboken, 2004, 264.

[2] Bronzino, J.D., ed., Medical Devices and Systems, $3^{\text {rd }}$ edn., Taylor \& Francis, London, 2006, 68-2.

[3] MDA London, Infusion Systems - Device Bulletin, DB 2003(02) March 2003, ISBN 1841826898.

[4] British Standards Institution, Medical electrical equipment - particular requirements for the safety of infusion pumps and controllers, BS EN 60601-2-24:1998, BS 5724-2.124:1998, IEC 60601-224: 1998 .

[5] Bath Institute of Medical Engineering, http://www.bath.ac.uk/bime/evalcentre/pumps.html, accessed Sept. 2009.

[6] Neff TA, Fellmann C, Fuechslin RM, Gerber AC, Weiss M (2002) The Panomat P-10 microvolumetric infusion pump is suitable for continuous drug administration at minimal flow rates. Can J Anaesth. 49: 1048-1052.

[7] Weiss M, Gerber S, Füchslin RM, Neff TA (2004) Accurate continuous drug delivery at low infusion rate with a novel microvolumetric infusion pump (MIVP): pump design, evaluation and comparison to the current standard. Anaesthesia. 59: 1133-1137.

[8] Murphy, R. S. and Wilcox, S. J., High risk infusions-accuracy compromised by changes in patient venous pressure, Journal of Medical Engineering \& Technology, 2009; 33:6,470-474.

[9] Krauskopf, K.H., Rauscher, J. and Brandt, L., Disturbance of continuous, pump administration of cardiovascular drugs by hydrostatic pressure, Der Anaesthesist, 1996 May; 45(5):449-52.

[10] Kern, H., Kuring, A., Redlich, U., Dopfmer, U.R., Sims, N.M., Spies, C.D. and Kox, W.J., Downward movement of syringe pumps reduces syringe output, Br J Anaesth, 2001; 86:828-31. 
[11] Neff, T.A., Fischer, J.E., Schulz, G., Baenziger, O. and Weiss, M., Infusion pump performance with vertical displacement: effect of syringe pump and assembly type, Intensive Care Med., 2001 Jan; 27(1):287-91.

[12] Weiss, M., Hug, M.I., Neff, T. and Fischer, J., Syringe size and flow rate affect delivery from syringe pumps, Can J Anaesth. 2000 Oct; 47(10):929-35.

[13] Murray, D., Another central line complication: failure of inotrope delivery due to equipment incompatibility, Anaesthesia, 2003; 58: 715.

[14] Neff, T.A., Fischer, J., Fehr, S., Banziger, O. and Weiss M., Start-up delays of infusion syringe pumps, Paediatr Anaesth, 2001; 11(5):561-565.

[15] Weiss M, Baenziger O, Neff T, Fanconi S (2000) Influence of infusion line compliance on drug delivery rate during acute line loop formation. Intensive Care Med. 26: 776-779.

[16] Weiss M, Neff T, Gerber AC, Fischer J (2000) Impact of infusion line compliance on syringe pump performance. Paediatr Anaesth. 10: 595-599.

[17] Fuechslin RM, Weiss M, Duenkel R, Mejer PF, Neff T, Stoll EP, Suter A. United States Patent Nr 7,614,857 B2 Nov 2009 Assignee: Medinnovation AG Zurich (CH). 



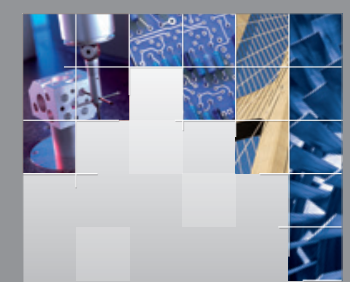

\section{Enfincering}
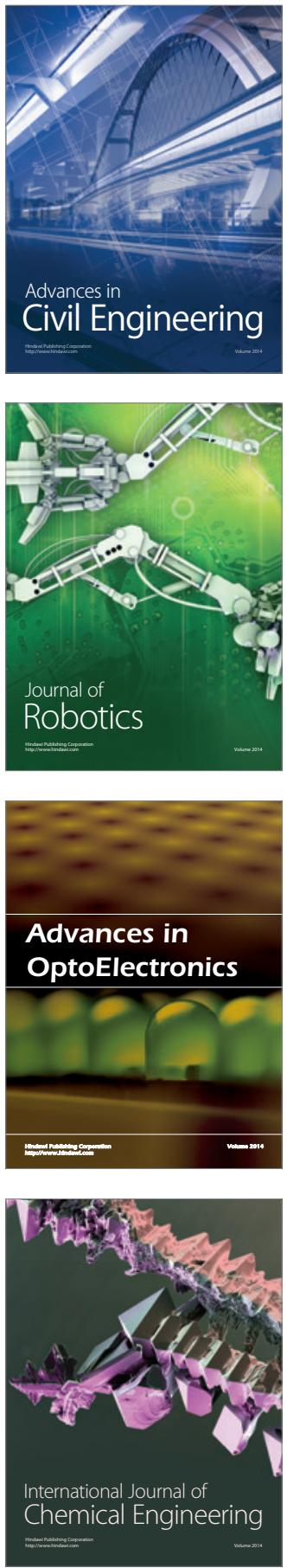

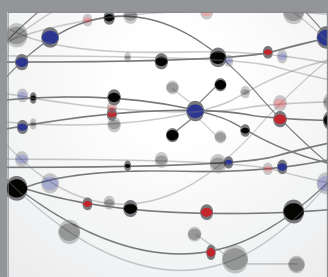

The Scientific World Journal

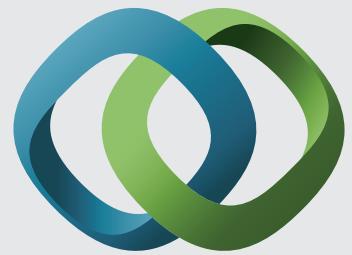

\section{Hindawi}

Submit your manuscripts at

http://www.hindawi.com
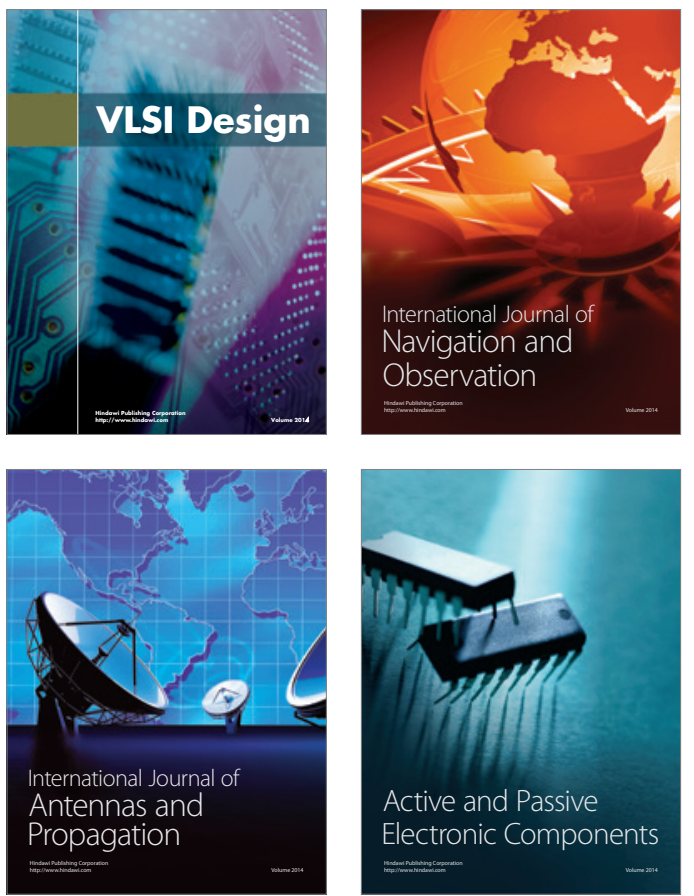
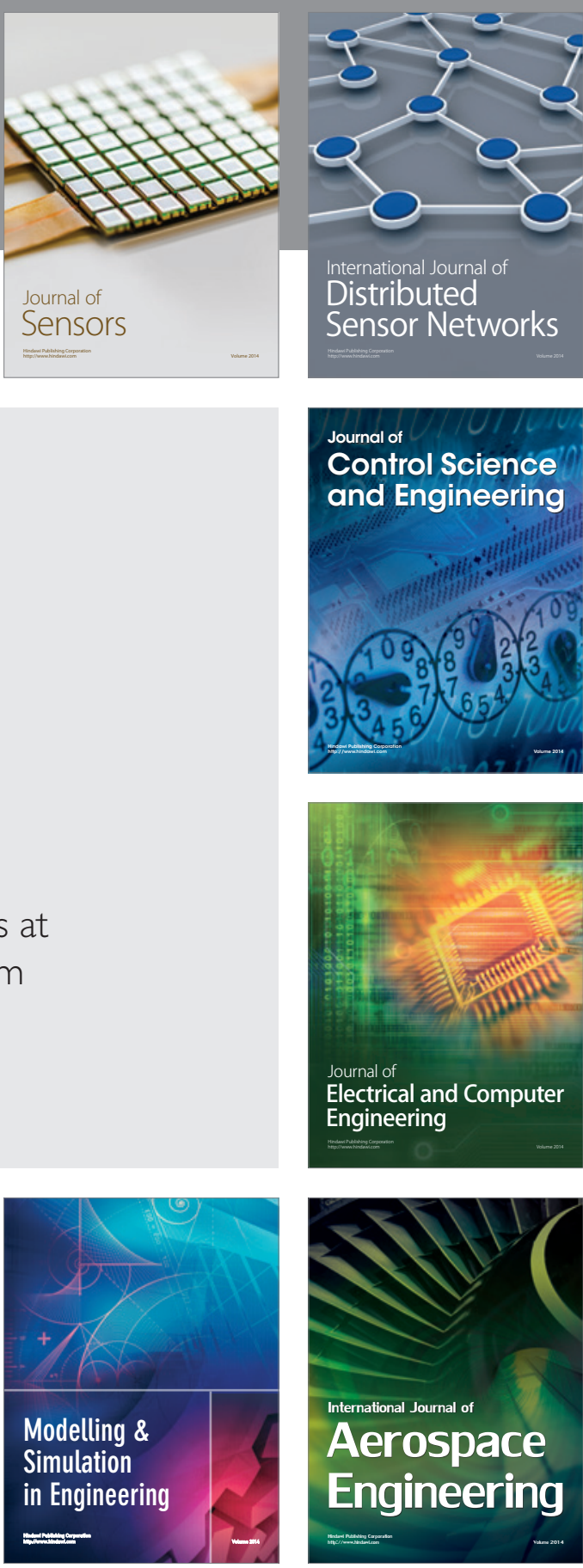

International Journal of

Distributed

Sensor Networks

Journal of

Control Science

and Engineering
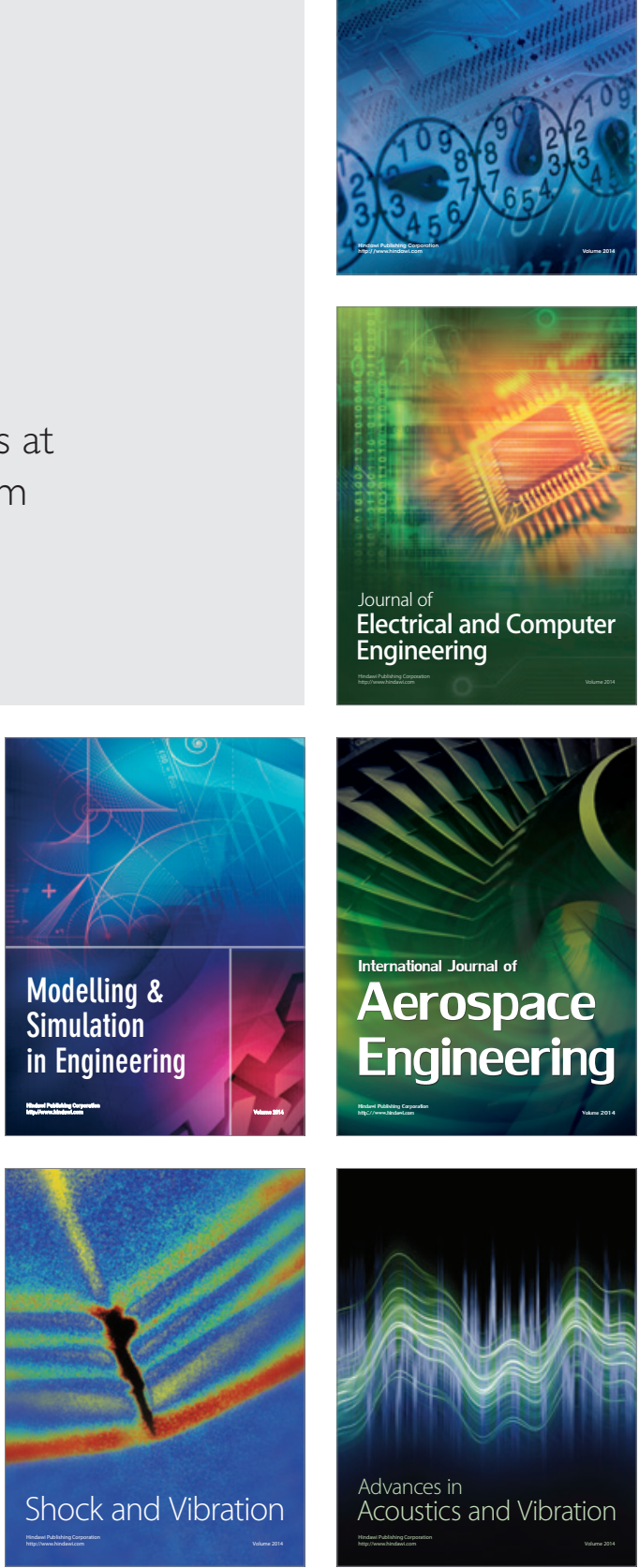